\title{
Depression, Sexual dysfunction and Quality of Life Among Breast Cancer Patients with Ovarian Function Suppression: A Cross Sectional Study between Ovarian Ablation Verse GnRH Agonists
}

junhan Jiang ( $\nabla$ jiangjunhan@outlook.com )

First affiliated Hospital of China Medical University

Junnan Xu

Cancer Hospital of China Medical University

Li Cai

Tumor Hospital of Harbin Medical University

Juan $\mathrm{Hu}$

Hunan Cancer Hospital

Li Man

Anshan Cancer Hospital

Limin Niu

Henan Cancer Hospital

Tao Sun

Cancer Hospital of China Medical University

Xinyu Zheng

First Affiliated Hospital of China Medical University

\section{Research}

Keywords: Breast cancer, ovarian function suppression, depression, sexual dysfunction, quality of life

Posted Date: August 28th, 2020

DOl: https://doi.org/10.21203/rs.3.rs-66195/v1

License: (c) (1) This work is licensed under a Creative Commons Attribution 4.0 International License. Read Full License 


\section{Abstract}

Background: Ovarian function suppression is being widely utilized as endocrine therapy to reduce estrogen release in premenopausal breast cancer patients and was achieved either by medical treatment with bilateral oophorectomy, irradiation, or the Gonadotropin releasing hormone (GnRH) agonist. This study aimed to examine whether GnRHa differed from ovarian ablation on depression, sexual dysfunction and quality of life.

Methods: The premenopausal breast cancer patients who received ovarian function suppression were enrolled from seven hospital between June 2019 and June 2020. Our independent variable was the type of ovarian suppression, categorized as Ovarian Ablation (OA cohort, $\mathrm{n}=174$ ) and medical $\mathrm{GnRH}$ agonist (GnRHa cohort, $n=389$ ). The self-administered questionnaire (OFS-Q5) was developed and used in this study aimed to assess the depression (PHQ-9), sexual dysfunction (FSFI) and quality of life (EORTC QLQBR23).

Results: In this cross-sectional study, 563 patients with ovarian function suppression completed surveys were collected. The mean sum score of the PHQ-9 tend to be slight decrease in GnRHa cohort than that in ovarian ablation $(\mathrm{OA})$ cohort ( $11.4 \pm 5.7$ vs. $12.8 \pm 5.8, \mathrm{OR}=1.910, P=0.079)$. Patients with major depression (PHQ-9 $\geqq 15)$ was indicated significantly fewer in GnRHa cohort (31.1\% vs $40.2 \%, P=0.025)$. The more surprising correlation is less patients with sexual dysfunction $(61.5 \%, \mathrm{FSFI} 23)$ in OA cohort, a remarkable increase in $\mathrm{GnRHa}$ cohort $(72.2 \%, P=0.011)$. The ratio of sexual dysfunction remained lower for ovarian ablation women in long-term ovarian suppression (duration of ovarian suppression $>2$ years: $\mathrm{OA}$ vs $\mathrm{GnRHa}$, $\mathrm{OR}=1.555, \mathrm{P}=0.037$ ). No significantly difference for most subscales of QLQ-BR23 between two cohorts was evident.

Conclusions: Our current investigation demonstrate here for the first time that medical GnRHa resulted in favour depression, worse sexual function than those with ovarian ablation, with similar quality of life. This new understanding should help to improve and alleviate adverse effect in patients with diverse ovarian function suppression.

\section{Introduction}

Breast cancer is the most commonly diagnosed cancer and the leading cause of cancer death among females worldwide, with a remarkable increase young breast cancer in China. [1] Premenopausal has been proved to represent worse clinical outcomes as an independent predictor in hormone-receptor (HR) positive breast cancer and more premenopausal patients with breast cancer diagnosed in China with the median age $45 \sim 55$ years, and approximately $6 \%$ of patients with breast cancer are less than 40 years old [2]. In previous decades, an extended adjuvant endocrine therapy with ovarian function suppression in premenopausal breast cancer patients, contributed to extra beneficial for HR-positive population [3]. Limited knowledge of the impact of ovarian function suppression on depression, sexual function and 
quality of life was indicated and compared in patients with ovarian ablation or gonadotropin releasinghormone $(\mathrm{GnRH})$ agonists until recently.

Ovarian function suppression (OFS) was the first adjuvant endocrine therapy investigations in premenopausal breast cancer patients and can eliminate or reduce estrogen level produced from ovarian. Initially, ovarian function was suppressed achieved by surgical bilateral oophorectomy or ovarian irradiation [4, 5], and more recently with medical $\mathrm{GnRH}$, (also named as luteinizing-hormone releasinghormone, LH-RH) agonists [6, 7]. Ovarian function suppression was defined as surgical bilateral oophorectomy, ovarian irradiation or $\mathrm{GnRH}$ agonists. Limited the ovarian irradiation for ovarian function suppression in real world in China, we only enrolled and compared surgical bilateral oophorectomy and $\mathrm{GnRH}$ agonists in our study. It is now well established that ovarian function suppression has the similar effect on reducing the risk of breast cancer relapse or distant metastases with previous CMF regime [8]. The suppression of ovarian function trial (SOFT) clinical trial reported that premenopausal women age < 35 years with high risk for relapse achieved a larger benefit from OFS combined with tamoxifen (freedom from breast cancer rate at 5 years, $78.9 \%$; 95\% $\mathrm{Cl}, 69.8-85.5 \%$ ) when compared with tamoxifen alone $(67.7 \% ; 95 \% \mathrm{Cl}, 57.3-76.0 \%)$, and the benefit was further enhanced by ovarian function suppression with exemestane (83.4\%; 95\% Cl, 74.9-89.3\%) [9-11]. There is an urgent need to address ovarian function suppression with $\mathrm{GnRHa}$ was achieved to protect ovarian function and improve infertility by chemotherapy $[12,13]$. A questionnaire to survey was performed in breast cancer patients younger than 35 years and $8 \%$ of patients rejected to receive chemotherapy because of worried about reducing their fertility [14]. GnRHa can protect ovarian function during chemotherapy for HR-negative breast cancer patients [15]. More important for clinicians and patients were to understand the state of ovarian function and protect their ovarian function. Ovarian function suppression is being widely utilized as endocrine therapy to reduce estrogen release in premenopausal early and metastatic breast cancer patients $[16,17]$ or as ovarian protector in premenopausal early breast cancer patients prior to adjuvant chemotherapy [18].

Ovarian function suppression in SOFT study was achieved either by medical treatment with the GnRHa triptorelin, bilateral oophorectomy, or ovarian irradiation. A comprehensive analysis of SOFT demonstrated that ovarian suppression with $\mathrm{GnRHa}$ has a similar effect to ovarian ablation with remarkable advantages in premenopausal women $[9,11]$. Firstly, $\mathrm{GnRHa}$ can be stopped as a reversible medicine if the patient experiences intolerable symptoms, thus GnRHa may be preferred over permanent ovarian ablation with bilateral oophorectomy or ovarian irradiation. All ovarian function suppression contributed diverse premature ovarian failure with significant consequences, including infertility, sexual dysfunction and vasomotor symptoms [19]. Young survivors consider premature menopause, sexual dysfunction and infertility to represent the most distressing aspects of their breast cancer experience [20]. So far, however, there has been little discussion about emotional disorder (depression), sexual dysfunction and poor quality of life with different ovarian function suppression strategies. This study attempts to examine whether GnRHa was superior to ovarian ablation with lower major depression, sexual dysfunction and worse quality of life in premenopausal patients with breast cancer and finally, to 
investigate whether social demographic and clinical characteristics can predict high risk for major depression, sexual dysfunction and worse quality of life during ovarian function suppression.

\section{Methods}

\section{Participants}

An anonymous, cross-sectional study was collected from 350 women accessed as a convenience sample between July 2019 and June 2020 from 7 hospitals in China. Five hundred and sixty-three eligible patients underwent ovarian ablation (bilateral oophorectomy) or gonadotropin- releasing hormone $(\mathrm{GnRH})$ agonists [goserelin acetate (Zoladex, AstraZeneca) at the dose of $3.6 \mathrm{mg}$ once every month, or leuprorelin acetate (Leuplin, Takeda) at the dose of $3.75 \mathrm{mg}$ once every month or $11.25 \mathrm{mg}$ once every three month] by intramuscular injection for ovarian function suppression. Limited to the ovarian irradiation for ovarian function suppression in real world in China, we only included surgical bilateral oophorectomy and $\mathrm{GnRH}$ agonists in our study and the patients were excluded with bilateral ovarian irradiation in our study. Eligible subjects had to be Chinese-speaking aged from 18 to 65 years and completed the questionnaire.

Patients were invited to complete electronic questionnaires labeled Breast Cancer Survivorship Ovarian Function Suppression Survey OFS-Q5 including PHQ-9, FSFI and QLQ-BR23. The validity and reliability of these Chinese version questionnaires have been previously proven [21-23]. Anonymity was added to the study to improve participation and response accuracy. The implied consent was described in the cover letter. Participants fitting the following criteria were included in our study: 1) clearly diagnosed with breast cancer (tissue or cell diagnosis), 2) age more than 18 years old, 3) received ovarian function suppression with surgical bilateral oophorectomy or $\mathrm{GnRH}$ agonists. The exclusion criteria were as follows: 1) being unwilling to give an informed consent, 2) being unable to understand the questionnaires, or 3) ovarian irradiation for ovarian function suppression. A written informed consent was obtained from each participant and the study protocols were reviewed and proved by the Ethic Committee of Cancer Hospital of China Medical University (protocol number RB \#20190545). The study was conducted in accordance with the Declaration of Helsinki.

\section{Questionnaire and Date measurement}

The self-administered questionnaire (OFS-Q5) developed and used in this study aimed to assess the depression, sexual dysfunction and quality of life in breast cancer patients with OFS between Ovarian Ablated (OA) and $\mathrm{GnRH}$ agonist (GnRHa), as well as the contributing demographic and clinical factors. The final questionnaire was divided into five sections. The section I comprised mostly society demographic and clinical characteristics, whereas the second section recorded ovarian function suppression information, and the following three sections (section from III to $\mathrm{V}$ ) evaluated depression, sexual function and quality of life in both cohorts. The final form of the questionnaire was approved by 
all investigators. Two survey assistants were trained the knowledge and communication skills regarding the ovarian function suppression and breast cancer. The first assistant helped to confirm and explain the information step by step beside the patient, and the second assistant re-confirm the answers by telephone $3 \sim 10$ days after survey. It was written in Chinese, the native language of the participants.

The society-demographic characteristics collected were as follows: age, educational level (low: high school or below), annual income (low: 50,000 RMB or less), smoking habit (never or little: smoking index of less than 200; the average root number per day multiplied by smoking years, that is smoking index), alcohol drinking habit (never or little: beer less than $2500 \mathrm{~mL}$ or spirit less than $500 \mathrm{~mL}$ ), co-morbidity (diabetes and/or hypertension, or none). The clinical data collected were as follows: type of surgery, tumor size, node, metastasis (TNM) staging classification, ER/PR/HER2/Ki67 status. The OFS section information were collected including the type of OFS (OA or GnRHa), time since OFS, adjuvant endocrine therapy, endocrine therapy duration and whether you choose the other type of OFS with or without the cost.

\section{Depression Questionnaire- PHQ-9}

Depression in patients with ovarian function suppression was quantified and assessed by utilizing the Chinese translation of Patient Health Questionnaire (PHQ-9), a 9-items survey in Chinese. The PHQ-9 is a 9-item screening instrument. Each of the 9 items received a score of 0-3. The following PHQ-9 cut-off scores have been recommended to determine levels of depression severity: 0-7 none and/or mild, 8-14 moderate, 15-19 moderate to severe, and 20-27 severe. Lower scores correspond with better emotion and major depression was defined as a sum score of $\geq 15$.

\section{Sexual Function Questionnaire- FSFI}

Sexual function was quantified by utilizing the FSFI, a 19-items survey instrument that specifically assessed six sexual functioning domains (FSFI 1-desire, FSFI 2-arousal, FSFI 3-lubrication, FSFI 4orgasm, FSFI 5-satisfaction, and FSFI 6-pain). Sexual activity was only assessed for the 4 weeks before the survey. This tool was developed based on the major categories of female sexual dysfunction as defined by the Diagnostic and Statistical Manual of Mental Disorders (DSM) and validated the FSFI in breast cancer survivors with credible reliability $(>0.9)$ and validity $(>0.8)$. Lower scores correspond with worse sexual function, and an overall score of less than 23 was defined as sexual dysfunction. To detect differences among cohorts based on the type of ovarian function suppression (OA verse GnRHa), FSFI total scores and each sub-item score were compared among the two groups with mean and median algorithms.

\section{Quality of Life Questionnaire- EORTC QLQ-BR23}


Quality of life was assessed by completing the European Organization for the Research and Treatment of Cancer - Breast Cancer quality of life 23 items Form (EORTC QLQ-BR23) and a breast cancer-specific module consisted of two core sections and 8 sub-items including (body image, sexual functioning, sexual enjoyment and future perspective in functional scales; systemic therapy side effects, breast symptoms, arm symptoms, upset by hair loss in symptom scales). All indicators were in linear analog selfassessment format and transformed to range from 0 to 100 by standardization, with higher scores reflecting a better condition.

\section{Statistical methods}

Data analysis was performed with the statistical package SPSS software version 23.0 (IBM Inc., Armonk, NY, USA). Survey items that were left blank were excluded from the analysis of that particular question or reported as "unknown" for demographic data. Participants' characteristics and PHQ9/FSFI/BR23 scores were presented as percentages for categorical variables and mean \pm standard deviation for continuous variables. Fisher's exact test was used for categorical data. Differences in terms of age, duration of ovarian function suppression (OFS) were analyzed using unpaired t-tests. The mean of PHQ9 total scores, FSFI total scores, FSFI sub-scores and BR23 sub-scores non-normally distributed, non-parametric tests (Mann-Whitney $U$ test) were performed to determine. Binary logistic regression and multiple regression models were used to examine the effects of the type of ovarian function suppression on different domains of PHQ9/FSFI/BR23 after adjust for age, duration of OFS, education, annual income, smoking/alcohol drink habit, co-morbidity, type of surgery and tumor characteristics (tumor size, regional lymph node metastasis, distant metastasis), and HR/HER2 status. All statistical tests were two-sided, and $P$ values $<0.05$ were considered to indicate statistical significance.

\section{Results}

\section{Patient characteristics}

In this cross-sectional investigation, total 591 patients were invited and 563 patients with ovarian function suppression completed surveys were collected for an estimated response rate of $95 \%(563 / 591)$. Twenty-eight surveys were excluded from the analysis for conflicting entries information of tumor or demographic characteristics. As shown in Fig. 1, the final group of patients with complete demographics characteristics and PHQ-9/FSFI/EORTC QLQ-BR23 scores consisting of 563 individuals. According to the type of ovarian function suppression, 174 patients with ovarian ablation for ovarian function suppression were in OA cohort and 389 patients with $\mathrm{GnRH}$ agonist were in $\mathrm{GnRHa}$ cohort. Table 1 illustrates the main characteristics between $\mathrm{GnRHa}$ and OA cohorts. The median ages of the female breast cancer survivors were 46.0 years in $\mathrm{OA}$ cohort and 43.0 years in $\mathrm{GnRHa}$ cohort $(P=0.013)$. At the time of survey response, what is striking in Table 1 is that time interval from ovarian suppression was significantly longer in $\mathrm{OA}$ cohort $(30.3 \pm 31.2$ months vs. $22.8 \pm 22.2$ months, $P=0.009)$. The majority of those response to this survey were low annual income (77.0\%), never or little smoking habit $(98.4 \%)$, never or little alcohol 
drinking habit (99.1\%), none co-morbidity (93.8\%), mastectomy surgery $(77.3 \%)$, stage I-III (73.3\%), estrogen receptor (ER) or progesterone receptor (PR)-positive (97.2\%), and HER2-negative (76.1\%). In addition, $59.2 \%$ of the patients in GnRHa cohort were educated up to college level of above and the percentage was only $42.4 \%$ in OA cohort. Interestingly, patients received medicine GnRHa were incline to breast conserving surgery $(26.0 \%$ vs $16.7 \%, P=0.015)$. 
Table 1

Baseline social demographics and clinical characteristics in breast cancer patients with ovarian function suppression

\begin{tabular}{|c|c|c|c|c|}
\hline Characteristics & Total & $\begin{array}{l}\text { Ovarian Ablation } \\
\text { OA }\end{array}$ & $\begin{array}{l}\text { GnRH agonist } \\
\text { GnRHa }\end{array}$ & P Value \\
\hline $\mathrm{N}$ & 563 & 174 & 389 & \\
\hline \multicolumn{5}{|l|}{ Age, years } \\
\hline Median & 44 & 46 & 43 & \\
\hline Range & $22-63$ & $31-61$ & $22-63$ & \\
\hline Mean (SD) & $42.7 \pm 7.8$ & $45.2 \pm 7.0$ & $41.6 \pm 7.8$ & 0.013 \\
\hline \multicolumn{5}{|l|}{ Mean (SD), months } \\
\hline Educational level & & & & 0.0002 \\
\hline High school or below & 268 & 103 & 165 & \\
\hline College or above & 295 & 71 & 224 & \\
\hline Annual income (RMB) & & & & 0.360 \\
\hline$\leq 50,000$ & 398 & 126 & 272 & \\
\hline$>50,000$ & 165 & 48 & 117 & \\
\hline Smoking habit & & & & 0.439 \\
\hline Never or little & 548 & 168 & 380 & \\
\hline Mostly & 15 & 6 & 9 & \\
\hline Alcohol drinking habit & & & & 0.271 \\
\hline Never or little & 556 & 170 & 386 & \\
\hline Mostly & 7 & 4 & 3 & \\
\hline Co-morbidity & & & & 0.077 \\
\hline Diabetes/Hypertension & 51 & 22 & 29 & \\
\hline None & 522 & 162 & 360 & \\
\hline Type of Surgery & & & & 0.015 \\
\hline Mastectomy & 433 & 145 & 288 & \\
\hline Breast-conserving & 130 & 29 & 101 & \\
\hline
\end{tabular}




\begin{tabular}{|c|c|c|c|c|}
\hline Characteristics & Total & $\begin{array}{l}\text { Ovarian Ablation } \\
\text { OA }\end{array}$ & $\begin{array}{l}\text { GnRH agonist } \\
\text { GnRHa }\end{array}$ & P Value \\
\hline \multicolumn{4}{|l|}{ TNM staging } & \multirow[t]{3}{*}{0.006} \\
\hline HIII & 400 & 110 & 290 & \\
\hline IV & 163 & 64 & 99 & \\
\hline \multicolumn{4}{|c|}{ Hormone Receptor Status } & \multirow[t]{3}{*}{0.0004} \\
\hline ER or PR Positive & 526 & 153 & 373 & \\
\hline ER and PR Negative & 37 & 21 & 16 & \\
\hline \multicolumn{4}{|l|}{ HER2 Status } & \multirow[t]{3}{*}{0.262} \\
\hline Positive & 130 & 35 & 95 & \\
\hline Negative & 433 & 139 & 294 & \\
\hline
\end{tabular}

\section{Major depression in GnRHa and OA cohorts}

Closer inspection of Table 2 shows mean PHQ-9 sum score in GnRHa cohort tend to be lower than that in ovarian ablation $(\mathrm{OA})$ cohort ( $11.4 \pm 5.7$ vs. $12.8 \pm 5.8, \mathrm{P}=0.079)$. According to the algorithm for depression [moderate (score 8-14), major (score 15-19), and severe (score 20-27) ], 36.2\% (63/174), $28.2 \%(49 / 174)$, and $12.6 \%(22 / 174)$ of patients were categorized in the moderate, major, and severe depression groups in OA cohort, and 42.9\% (167/389), 22.4\% (87/389), and 8.7\% (34/389) of patients were categorized in the moderate, major, and severe depression groups in GnRHa cohort, respectively. Noticeably, patients with major depression, defined by PHQ-9 $\geqq 15$, was indicated significantly fewer in GnRHa cohort than those in OA cohort. A positive correlation was revealed between major depression and ovarian ablation, whereas the absolute difference was approximately $9.1 \%(31.1 \%$ vs. $40.2 \%, P=0.025)$. Suicidal ideation was considered as a symptom of major depression. On the item level, 15 out of the 174 patients with ovarian ablation and 30 out of the 389 patients with $\mathrm{GnRHa}$ claimed to have suicidal ideation ( $8.6 \%$ vs. $7.7 \%, P=0.713$ ). What is striking about Table 3 is ovarian suppression type as the independent risk factor on major depression in uni-variate and multivariate analysis [Ovarian ablation vs GnRHa: $\operatorname{Exp}(B)=2.483$ (95\% Cl: $1.056 \sim 6.450), P=0.026$ ]. As mentioned above, patients received medicine $\mathrm{GnRHa}$ were incline to breast conserving surgery ( $26.0 \%$ vs $16.7 \%, \mathrm{P}=0.015)$, turning now to the investigate the relationship, however, data from Table 3 , it is apparent that the type of surgery was not the independent factor in multivariate analysis on major depression. Further analysis showed that the duration of ovarian function suppression was closely correlated with major depression, and there has been a remarkable time-dependent tendency [duration of OFS $>2$ year vs duration of OFS $\leq 2$ year : Exp $(B)=1.651$ (95\% Cl: $1.071 ~ 2.518), P=0.031]$ 
Table 2

Depression (PHQ-9) by the type of ovarian function suppression in patients with breast cancer

\begin{tabular}{|c|c|c|c|}
\hline & \multicolumn{3}{|c|}{ Ovarian function suppression } \\
\hline & Ovarian Ablation (OA) & GnRH agonist (GnRHa) & $P$ value \\
\hline & $\mathrm{n},(\%)$ & $\mathrm{n},(\%)$ & \\
\hline Total & 174 & 389 & \\
\hline PHQ-9 scores Median & 13 & 11 & \\
\hline (IQR) & $(8.0-17.0)$ & $(7.0-16.0)$ & \\
\hline PHQ-9 scores Range & $1-24$ & $0-25$ & \\
\hline PHQ-9 scores Mean (SD) & $12.8 \pm 5.8$ & $11.4 \pm 5.7$ & 0.079 \\
\hline \multicolumn{4}{|l|}{ PHQ-9 scores subgroups } \\
\hline None or mild $(0-7), \mathrm{n}(\%)$ & $40(23.0)$ & $101(26.0)$ & \\
\hline Moderate (8-14), n(\%) & $63(36.2)$ & $167(42.9)$ & \\
\hline Major (15-19), n(\%) & $49(28.2)$ & $87(22.4)$ & \\
\hline Severe (20-27), n(\%) & $22(12.6)$ & $34(8.7)$ & \\
\hline Major Depression & 71 & 121 & 0.025 \\
\hline$(\mathrm{PHQ} \geq 15), \mathrm{n}(\%)$ & $(40.2)$ & $(31.1)$ & \\
\hline Suicidal ideation, n(\%) & $15(8.6)$ & $30(7.7)$ & 0.713 \\
\hline
\end{tabular}


Table 3

The associated factors on major depression (PHQ-9, scores $<15$ vs $\geq 15$ ) by logistic regression in patients with breast cancer

\begin{tabular}{|c|c|c|c|c|c|c|}
\hline \multirow[t]{2}{*}{ Characteristics } & \multicolumn{3}{|c|}{ Univariate } & \multicolumn{3}{|c|}{ Multivariate } \\
\hline & $\mathrm{OR}$ & $95 \% \mathrm{Cl}$ & $P$ value & OR & $95 \% \mathrm{Cl}$ & $P$ value \\
\hline \multicolumn{7}{|l|}{ Age, years } \\
\hline$\leq 45$ & 1 & $0.924-2.095$ & 0.113 & & & \\
\hline$>45$ & 1.392 & & & & & \\
\hline \multicolumn{7}{|l|}{ Educational level } \\
\hline High school or below & 1 & $0.522-1.145$ & 0.199 & & & \\
\hline College or above & 0.773 & & & & & \\
\hline \multicolumn{7}{|l|}{ Annual income (RMB) } \\
\hline$\leq 50,000$ & 1 & $0.577-1.351$ & 0.565 & & & \\
\hline$>50,000$ & 0.883 & & & & & \\
\hline \multicolumn{7}{|l|}{ TNM staging } \\
\hline I- III & 1 & $0.641-2.373$ & 0.530 & & & \\
\hline IV & 1.233 & & & & & \\
\hline \multicolumn{7}{|l|}{ Type of OFS } \\
\hline GnRH agonist & 1 & $1.006-2.518$ & 0.043 & 2.483 & $1.056-6.450$ & 0.026 \\
\hline Ovarian Ablation & 1.910 & & & & & \\
\hline \multicolumn{7}{|l|}{ Type of Surgery } \\
\hline Breast-conserving & 1 & $1.338-3.230$ & 0.001 & 1.459 & $0.951-3.630$ & 0.058 \\
\hline Mastectomy & 2.079 & & & & & \\
\hline \multicolumn{7}{|l|}{ OFS Time } \\
\hline$\leq 2$ years & 1 & $1.010-1.568$ & 0.037 & 1.651 & $1.071-2.518$ & 0.031 \\
\hline$>2$ years & 1.555 & & & & & \\
\hline \multicolumn{7}{|l|}{ HER2 Status } \\
\hline Negative & 1 & $0.420-1.283$ & 0.278 & & & \\
\hline Positive & 0.734 & & & & & \\
\hline
\end{tabular}




\begin{tabular}{|c|c|c|}
\hline Characteristics & Univariate & Multivariate \\
\hline \multicolumn{3}{|c|}{ Endocrine Therapy } \\
\hline $\mathrm{Al}$ & $0.655-1.619$ & 0.898 \\
\hline TAM & 1.030 & \\
\hline
\end{tabular}

\section{Sexual dysfunction in GnRHa and OA cohorts}

With successive diverse affect on major depression, we moved further to investigate whether varied sexual dysfunction between GnRHa and OA methods. Patients received GnRHa had lower mean and median FSFI scores compared with patients with ovarian ablation (OA), as indicated in Table 4 (mean \pm SD: $17.8 \pm 8.7$ vs $19.3 \pm 8.5, P=0.205$; median: 17.8 vs 19.6 ), only trace difference amounts of mean FSFI sum scores were detected. If we turn to the sexual dysfunction, defined as $\mathrm{FSFI}<23$, strong evidence of GnRHa- induced sexual dysfunction was revealed, more than half of patients with ovarian ablation met the criteria for sexual dysfunction $(61.5 \%, \mathrm{FSFI}<23)$ compared with $72.2 \%$ of patients with $\mathrm{GnRHa}(\mathrm{P}=$ 0.011). From the data in Fig. 2, patients with GnRHa had a little lower most of sub-FSFI scores than patients with ovarian ablation but remained a clear significantly lower in pain section (FSFI-6 scores: mean $\pm S D: 3.3 \pm 2.2$ vs $2.5 \pm 2.2, P=0.007$ ) and significantly difference in lubrication section (FSFI-3 scores: mean \pm SD: $3.5 \pm 2.5$ vs $2.9 \pm 2.6, P=0.048$ ). T-tests revealed no significant differences in mean scores on sexual desire (FSFI-1) and arousal (FSFI-2), in contrast to other sub-items, sexual orgasm and sexual satisfaction was more benefit in GnRHa cohort but the difference could not be identified in this analysis. Data from the uni-variate and multivariate analysis, a positive correlation between diverse ovarian function suppression type and sexual dysfunction was revealed (GnRHa vs OA: OR $=1.960,95 \%$ Cl: $1.070 \sim 3.591, P=0.029)$. In uni-variate analysis, age received OFS, educational level and combined endocrine therapy were the significantly risk factors for sexual dysfunction, and further multivariate analysis were evaluated. Adjusting for other factors, age received OFS and educational level remained lower odd ratio (Age $>45$ years vs Age $\leq 45$ years, $\mathrm{OR}=0.587,95 \% \mathrm{Cl}: 0.356 \sim 0.967, \mathrm{P}=0.037$; College or above vs High school or below: $\mathrm{OR}=0.433,95 \% \mathrm{Cl}$ : $0.258 \sim 0.726, \mathrm{P}=0.002)$. Despite of the limited samples for tamoxifen users, the associations were not found in multivariate analysis when compared combined endocrine therapy between tamoxifen users and aromatase inhibitor users $(\mathrm{OR}=1.160,95 \% \mathrm{Cl}$ : $0.310 \sim 4.341, P=0.826$ ). Further large sample research needs to be investigated the correlation between sexual dysfunction and combined endocrine therapy. 
Table 4

Sexual function by the type of ovarian function suppression in patients with breast cancer Ovarian function suppression

$\begin{array}{ll}\text { Ovarian Ablation (OA) } & \text { GnRH agonist (GnRHa) } \quad P \text { value } \\ \mathrm{N}=174 & \mathrm{~N}=\mathbf{3 8 9}\end{array}$

\section{FSFI total scores}

Mean (SD)

$19.3 \pm 8.5$

$17.8 \pm 8.7$

0.205

Median (IQR)

$19.6(13.0-26.7)$

$17.8(12.2-24.3)$

Range

2-34

2-34

Sexual dysfunction (FSFI < 23), n(\%)

$107(61.5)$

$281(72.2)$

0.011

FSFI, female sexual function index; SE, standard errors; IQR, interquartile range (25th, 75th percentiles). 
Table 5

The associated factors on sexual dysfunction ( $F S F I$, scores $\geq 23$ vs $<23$ ) by logistics regression in patients with breast cancer

\begin{tabular}{|c|c|c|c|c|c|c|}
\hline \multirow[t]{2}{*}{ Characteristics } & \multicolumn{3}{|c|}{ Univariate } & \multicolumn{3}{|c|}{ Multivariate } \\
\hline & OR & $95 \% \mathrm{Cl}$ & $P$ value & OR & $95 \% \mathrm{Cl}$ & $P$ value \\
\hline \multicolumn{7}{|l|}{ Age, years } \\
\hline$\leq 45$ & 1 & $0.403-1.030$ & 0.066 & 0.587 & $0.356-0.967$ & 0.037 \\
\hline$>45$ & 0.645 & & & & & \\
\hline \multicolumn{7}{|l|}{ Educational level } \\
\hline High school or below & 1 & $0.341-0.891$ & 0.015 & 0.433 & $0.258-0.726$ & 0.002 \\
\hline College or above & 0.551 & & & & & \\
\hline \multicolumn{7}{|l|}{ Annual income (RMB) } \\
\hline$\leq 50,000$ & 1 & $0.560-1.533$ & 0.766 & & & \\
\hline$>50,000$ & 0.926 & & & & & \\
\hline \multicolumn{7}{|l|}{ TNM staging } \\
\hline I- III & 1 & $0.405-1.881$ & 0.728 & & & \\
\hline IV & 0.872 & & & & & \\
\hline \multicolumn{7}{|l|}{ Type of OFS } \\
\hline Ovarian Ablation & 1 & $1.041-3.244$ & 0.036 & 1.960 & $1.070-3.591$ & 0.029 \\
\hline GnRH agonist & 1.838 & & & & & \\
\hline \multicolumn{7}{|l|}{ Type of Surgery } \\
\hline Breast-conserving & 1 & $0.518-1.558$ & 0.703 & & & \\
\hline Mastectomy & 0.898 & & & & & \\
\hline \multicolumn{7}{|l|}{ OFS Time } \\
\hline$\leq 2$ years & 1 & $0.489-1.236$ & 0.287 & & & \\
\hline$>2$ years & 0.777 & & & & & \\
\hline \multicolumn{7}{|l|}{ HER2 Status } \\
\hline Negative & 1 & $0.764-3.844$ & 0.191 & & & \\
\hline Positive & 1.714 & & & & & \\
\hline
\end{tabular}

Al, aromatase inhibitors; FSFI, female sexual function index; OFS, ovarian function suppression; TAM, tamoxifen. 


\section{Characteristics}

Endocrine Therapy

$\mathrm{Al}$

TAM

$1.249-4.456$

0.008

Al, aromatase inhibitors; FSFI, female sexual function index; OFS, ovarian function suppression; TAM, tamoxifen.

\section{Quality of Life in GnRHa and OA cohorts}

The next section of the survey was concerned with quality of life. Mean scores of breast cancer patients with GnRHa in only one QLQ-BR23 functional dimensions, future perspective (BRFU). What stands out in Table 6 is a positive correlation between future perspective and GnRHa (GnRHa vs. OA: BRFU, $77.0 \pm 31.6$ verse $70.3 \pm 32.0, P=0.039$ ). This table is quite revealing in several ways. Firstly, poor sexual functioning and enjoyment in dimensions BRSEF and BRSEE were revealed with similar tendency in FSFI scores (GnRHa vs. OA: BRSEF, $55.9 \pm 37.9$ verse $61.7 \pm 37.8, \mathrm{P}=0.208$; BRSEE, $35.0 \pm 35.1$ vs. $38.7 \pm 36.6, \mathrm{P}=$ 0.421). More patients suffered side effects (BRST), breast symptoms (BRBS), arm symptoms (BRAS) and upset by hair loss (BRHL) in ovarian ablation (OA) cohort, but no significant $(P=0.502 ; P=0.310 ; \mathrm{P}=$ $0.512 ; P=0.785$ ). This is an expected result, EORTC QLQ-BR23 focused on the breast cancer-related symptom and function, but not only regarding ovarian function, the differences of most sub-scales between $\mathrm{OA}$ and $\mathrm{GnRHa}$ were insignificant. 
Table 6

Quality of Life Scores (EORTC-BR23) according to the type of ovarian function suppression in patients with breast cancer

EORTC-BR23 scores

$($ mean \pm SD)

\section{Ovarian function suppression}

Ovarian Ablation

$N=174$
GnRH agonist

$P$ value

\section{Functional scales}

\begin{tabular}{lllll} 
Body image & BRBI & $68.0 \pm 25.8$ & $72.3 \pm 24.0$ & 0.190 \\
\hline Sexual functioning & BRSEF & $61.7 \pm 37.8$ & $55.9 \pm 37.9$ & 0.208 \\
\hline Sexual enjoyment & BRSEE & $38.7 \pm 36.6$ & $35.0 \pm 35.1$ & 0.421 \\
Future perspective & BRFU & $70.3 \pm 32.0$ & $77.0 \pm 31.6$ & 0.039
\end{tabular}

\section{Symptom scales}

$\begin{array}{lllll}\text { Side effects } & \text { BRST } & 54.4 \pm 16.3 & 52.9 \pm 15.0 & 0.502 \\ \text { Breast symptoms } & \text { BRBS } & 32.8 \pm 26.7 & 34.5 \pm 22.9 & 0.310 \\ \text { Arm symptoms } & \text { BRAS } & 36.6 \pm 25.3 & 37.7 \pm 23.0 & 0.512 \\ \text { Upset by hair loss } & \text { BRHL } & 90.5 \pm 21.7 & 89.6 \pm 23.2 & 0.785\end{array}$

BRBI, body image; BRSEF, sexual functioning; BRFU, future perspective; BRSEE, sexual enjoyment; BRST, systemic therapy side effect; BRBS, breast symptoms; BRAS, arm symptoms; BRHL, upset by hair loss.

\section{Replacement ovarian function suppression}

In the final part of our survey, respondents were asked "Would you like to choose the other ovarian function suppression, for example, would you like to choose ovarian ablation instead of medicine $\mathrm{GnRHa}$ and vice versa? " Surprisingly, the majority of both participants considered this alternation. Approximately $62.4 \%$ patients chose to exchange the type of ovarian function suppression ( $\mathrm{GnRHa}$ : $62.7 \%$ vs OA: $60.8 \%$ ). Concerns regarding costs may affect the final choice, we also asked another question "Without considering the cost and economy, would you like to choose the other ovarian function suppression, for example, would you like to choose ovarian ablation instead of medicine GnRHa and vice versa?" This is an expected outcome, without taking into account of medical-related economic cost, the exchange ratio greatly increased and was up to $70.3 \%$ in ovarian ablation cohort, whereas, no significantly increased in GnRHa cohort (67.1\%). However, the observed difference between ovarian ablation and GnRHa in our study was not significant, the absolute differences of replacement were $3.2 \%$ in ovarian ablation and only $1.5 \%$ in patients with GnRHa. The majority of both cohorts may be unsatisfied the current ovarian function suppression regarding depression, sexual dysfunction and worse quality of life, and the majority of participants were reluctant to receive ovarian ablation limited to the medical-related economic costs. 


\section{Discussion}

A cross-sectional investigation to explore the association between depression, sexual dysfunction, quality of life and the type of ovarian function suppression (ovarian ablation and medical GnRH agonist) among breast cancer patients in our study. The results demonstrated that GnRHa presented clinical meaningful lower major depression and higher sexual dysfunction than that in ovarian ablation cohort, however, the domains of quality of life were small with differences in both ovarian function suppression treatments.

Major depression results correspond with our hypotheses and confirm previous findings [21, 24]. Young breast cancer survivors are the group most at risk for major depression problems due to abrupt menopause and breast disease and the ratio of major depression are greatly higher than would be expected in a community at the same age range. A meta analysis reported that the difference was insignificant of depression between ovarian function suppression (OFS) and non-ovarian function suppression in 3373 patients (RR $1.28,95 \% \mathrm{Cl}: 0.94-1.74, \mathrm{P}=0.12$ ), with no significant heterogeneity among four studies $\left(P=0.46, \mathrm{I}^{2}=0 \%\right)$ [25]. In contrast to this meta-analysis, we found that depression was reported as CTC AE grades not PHQ-9 scores in our study. For instance, Moore et al demonstrated that 8 cases with grade 2 and only 1 cases with grade 3 in OFS cohort and only three grade 2 in non-OFS cohort as adverse effect in early breast cancer patients [12]. In our investigation, we focused on major depression in early and metastatic breast cancer, the patients with metastatic breast cancer seriously suffered from major depression and the ratio of major depression was highlighted. Depression, linked to both the psychological aspects of society relationship and the physical effects of chemotherapy, which always can be overwhelming during metastatic treatment [26]. Longer-term ovarian function administration of a GnRH agonist was complicated assessed whereas the combination of adjuvant endocrine therapy and ovarian function suppression with synchronous or sequential chemotherapy. Depression was diminished after 2 years with duration of medical GnRHa and were no longer clinically meaningful compared without GnRHa treatment. However, the findings of the current investigation is contrary to the previous study. Major depression was gradual increased after 2 years with ovarian suppression, whereas patients with ovarian ablation suffered with higher major depression than that with medical GnRH agonist. To further investigation the potential mechanism of major depression with ovarian function suppression. Pooley $A$ et al reported that female rats with traumatic stress (depression model) was suggested a unique phenotype without HPA negative feedback but shown in male rats. A possible explanation for these results may be traumatic-related stress was largely independent of adult circulating gonadal hormone status and robust sex differences (female-biased) [27, 28]. Furthermore, circulating hormone and metabolism were highly correlated with major depression in premenstral dysphoric disorder (PMDD). Decreased pregnenoline and DHEA-sulfate was co-morbid with estrogen addback compared with lupron for increasing the accuracy of circulating diagnosis [29]. Co-morbid diabetes, hypertension, and coronary heart disease are accompanied by a high incidence of depression and can affect prognosis and compliance [30-31]. The limited cases with reported co-morbidity was inadequate to examine its effect on depression, sexual and quality of life in our study. 
In addition to the significant sexual dysfunction issues outlined above, ovarian function suppression and its side effects can contribute to reduced sexual desire and decreased sexuality. Strategies to reduce estrogen level might increase vaginal dryness and dyspareunia during the administration of GnRHa in premenopausal patients [32]. Aromatase inhibitors have the similar effects on sexual dysfunction with $\mathrm{GnRHa}$, however, aromatase inhibitors were not frequently utilized in premenopausal without ovarian function suppression. Compared with ovarian ablation, the sexual dysfunction issues by medical $\mathrm{GnRH}$ agonists could be reversible when $\mathrm{GnRHa}$ is withdrawn, however the permanent ovarian failure caused by ovarian ablation or ovarian failure by chemotherapy [33]. The sexual dysfunction problem is convenient to reverse. Surprisingly, compared with ovarian ablation, our results reveals that sexual dysfunction become more common and serious in $\mathrm{GnRHa}$ cohort independent of duration of ovarian function suppression. The trend of sexual dysfunction in this investigation was similar to that presented by other researchers, gradual decreased in ovarian ablation group and slight declined in GnRHa cohort after 2 years since ovarian function suppression. Interesting, the average age was younger in patients with GnRHa. At this time point, the mean age was 47.4 years in ovarian ablation cohort, and more women may have reached menopausal in ovarian ablation cohort. Research on cancer care demonstrates low satisfaction related to sexual desire in young survivor and more likely to be distressed at the alternations in appearance [34]. This inconsistency may be due to the disequilibrium distribution of age among the patients in our study. A possible explanation for these results may be the age differences and the spontaneous degeneration of sexual desire. Previous publishes studies suggested tamoxifen associated with sexual dysfunction remained controversy in premenopausal patients [35, 36]. Mild and partial estrogen effect of tamoxifen actually buffered postmenopausal vaginal dryness and the surveys did demonstrate that sexual desire decreased in patients treated with tamoxifen. Limited to the small samples combined with tamoxifen, the results could not fully explain the similar effect on sexual dysfunction in patients with ovarian function suppression in our study.

The epidemiology data are rather controversial, and there is no general agreement about the effect on sexual dysfunction by breast surgery. Estrogen deprivation make penetration and intercourse dry and painful by ovarian suppression. Previous investigation suggests that women with mastectomies suffered less sexual problems than women received breast conservation or breast reconstruction [37]. In contrast to the findings, however, no significantly difference was detected in our exploratory study in that patients with breast conservation suffered similar sexual dysfunction. Younger survivors may focus on breast loss with mastectomy, on the other hand, the body feeling of attractiveness affected on the sexuality. In addition, psychological status also play a critical role in sexuality and the understanding and concerning help to improve sexual satisfaction with closer sexual partners [38]. Sexuality issues are complex for young cancer survivors and can be influenced by physical issues, such as vaginal dryness and dyspareunia and psychological issues. Surprisingly, patients with GnRH agonist suffered fewer major depression but more serious sexual dysfunction and not positively correlation between major depression and sexual dysfunction. General psychological depression presented correlated with higher rates of sexual dysfunction and a prospective investigation revealed women suffered serious depression and sexual issue at the time of peak during 2 years since breast surgery and largely decreased depression and 
continued most sexual problems particularly in younger breast cancer survivors. Asian ethnicity patients are less open to talk about sexuality and may be more vulnerable to dysfunction after breast cancer. Likewise, several studies reported patients expected sexual health care and discussion but clinicians mostly prefer to pay more attention to combat the disease due to the lack of adequate knowledge and busy clinics [39]. Sexual function has improved in breast cancer survivors with a one-time nursing intervention for African American survivors in a six-session couples sex therapy program [40]. Patientsclinicians communication regarding sexual health is critical and necessary routine assessment in breast cancer survivors with ovarian function suppression during treatment planning and at follow-up, independent of the type of ovarian suppression. The mechanism of decreased sexual desire by ovarian suppression may be relation with low levels of plasma testosterone and androgen deprivation in young survivors. Whereas, testosterone concentration was not a favor predictor for sexuality in breast cancer survivors and no largely benefit was found on sexual desire with the replacement of transdermal testosterone [41].

A major concern is, an important issue, whether the effect on quality of life is differed among ovarian ablation and medical GnRHa. Based on previous investigations, there is a strong possibility that quality of life was significantly decreased with ovarian function suppression with diverse sections, including hot flushes, fatigue, pain and so on [32]. Vasomotor symptoms was common and not life-threatening in patients with ovarian suppression and estrogen depletion, however, its related adversely effect resulted in early treatment discontinuation. Importantly, no differences of quality of life in our investigation selected sample of all women received ovarian suppression were observed directly compared ovarian ablation and medical GnRH agonist. As clinician approach management decisions, they prefer to consider the frequency and severity of symptoms and their impact in quality of life.

Despite the fact that a significant association between lower major depression, more sexual dysfunction and $\mathrm{GnRHa}$ in breast cancer patients was indicated in our investigation, due to the inherent limitations of our cross-sectional study design, the limitations of our study are obvious. First, given the anonymous survey, all treatment information was based on patient recall and could not be re-checked for accuracy by medical records. The reporting might not accuracy enough to evaluate based on patient's responses to the questionnaire. A truly prospective evaluation may provide additional information and limit recall bias. Secondly, the cross-sectional design limited cause-and-effect conclusions on major depression and sexual dysfunction. The reliability and validity was confirmed on three questionnaire in Chinese [21-23, 42-43], We cannot ignore the factor that social bias potentially existed because the questionnaire was delivered and retrieved. Two assistants confirmed the answers with patients face to face and by telephone to reduce the bias. Additionally, given the risk of recovery of ovarian function or insufficient ovarian suppression, we can not evaluate the sufficient ovarian suppression in medical GnRHa cohort in our study. Whether insufficient ovarian suppression affect major depression, sexual dysfunction and quality of life remains unclear. Despite its exploratory investigation, this study offers some insight into the individual adverse effect management of ovarian function suppression. 


\section{Conclusions}

Ovarian ablation resulted in worse depression, favour sexual function than those with medical GnRHa, with similar quality of life. Further research needs to be examine more closely the links between major depression and ovarian function suppression and far more attention need to paid on adverse effect in premenopausal breast cancer patients with ovarian function suppression, and thus routinely applying a personalized approach seems warranted.

\section{Declarations}

\section{Ethics approval and consent to participate}

This study was conducted in full conformance with the International Conference for Harmonization (ICH) E6 guideline for Good Clinical Practice and the principles of the Declaration of Helsinki. A written informed consent was obtained from each participant and the study protocols were reviewed and proved by the Ethic Committee of Cancer Hospital of China Medical University (protocol number RB \#20190545).

\section{Consent for publication}

Not applicable.

\section{Availability of data and materials}

We would not share the data and material used in this manuscript, because we need them for further research.

\section{Competing interests}

The authors declare that they have no conflict of interest.

\section{Funding}

This work was supported by the Natural Science Foundation of Liaoning Province (No. 20180551215), Key R \& D guidance plan of Liaoning Province (2019JH8/10300020), Key Research Project of Liaoning (grant number 2018225076), cSCO Project (Y-2019Genecast-019, Y-HR2018-362), Wujieping Project (320675018541) and LiaoNing Revitalization Talents Program (XLYC1907160).

\section{Authors' contributions}


XZ, and TS: Conceived and designed the experiments. JX, LC, LM, LN, JH performed the survey. JJ and JX analyzed the datas. JX wrote the paper. All authors read and approved the final manuscript and submission of this manuscript.

\section{References}

1. DeSantis CE, Ma J, Gaudet MM, et al. Breast cancer statistics, 2019. CA Cancer J Clin. 2019;69(6):438-51.

2. Fan L, Strasser-Weippl K, Li JJ, et al. Breast cancer in China. Lancet Oncol. 2014;15(7):e279-89.

3. Yeo W, Ueno T, Lin C, et al. Treating HR+/HER2 - breast cancer in premenopausal Asian women: Asian Breast Cancer Cooperative Group 2019 Consensus and position on ovarian suppression. Breast Cancer Res Treat. 2019;177:549-59.

4. Metwally IH, Hamdy O, Elbalka SS, et al. Oophorectomy as a Hormonal Ablation Therapy in Metastatic and Recurrent Breast Cancer: Current Indications and Results. Indian J Surg Oncol. 2019; 10(3):542-546.

5. Nourmoussavi M, Pansegrau G, Popesku J, et al. Ovarian ablation for premenopausal breast cancer: A review of treatment considerations and the impact of premature menopause. Cancer Treat Rev. 2017;55:26-35.

6. Regan MM, Fleming GF, Walley B, et al. Adjuvant Systemic Treatment of Premenopausal Women With Hormone Receptor-Positive Early Breast Cancer: Lights and Shadows. J Clin Oncol. 2019;37(11):862-6.

7. Sa-Nguanraksa D, Krisorakun T, Pongthong W, et al. Survival outcome of combined GnRH agonist and tamoxifen is comparable to that of sequential adriamycin and cyclophosphamide chemotherapy plus tamoxifen in premenopausal patients with early breast cancer. Mol Clin Oncol. 2019;11(5):51722.

8. Recchia F, Candeloro G, Rosselli M, et al Adjuvant Ovarian Suppression. High-dose Chemotherapy and Immunotherapy for Premenopausal Patients with High-risk Breast Cancer. Anticancer Res. 2015;35(12):6847-53.

9. Francis PA, Regan MM, Fleming GF, et al. SOFT Investigators; International Breast CancerStudy Group. Adjuvant ovarian suppression in premenopausal breast cancer. N Engl J Med. 2015;372(5):436-46.

10. Francis PA, Pagani O, Fleming GF, et al, SOFT and TEXT Investigators and the International Breast Cancer Study Group. Tailoring Adjuvant Endocrine Therapy for Premenopausal Breast Cancer. N Engl J Med. 2018;379(2):122-37.

11. Pagani O, Francis PA, Fleming GF, et al; SOFT and TEXT Investigators and International Breast Cancer Study Group. Absolute Improvements in Freedom From Distant Recurrence to Tailor Adjuvant Endocrine Therapies for Premenopausal Women: Results From TEXT and SOFT. J Clin Oncol. 2019; JC01801967. 
12. Moore HC, Unger JM, Phillips KA, et al. POEMS/S0230 Investigators. Goserelin for ovarian protection during breast-cancer adjuvant chemotherapy. N Engl J Med. 2015;372(10):923-32.

13. Blumenfeld Z. Fertility Preservation Using GnRH Agonists: Rationale, Possible Mechanisms, and Explanation of Controversy. Clin Med Insights Reprod Health. 2019;13:1179558119870163.

14. Senkus E, Gomez H, Dirix L, et al. Attitudes of young patients with breast cancer toward fertility loss related to adjuvant systemic therapies. EORTC study 10002 BIG 3-98. Psycho-oncology. 2014; 23(2):173-182.

15. Allaway HC, M,Chizen DR, Adams GP, et al. Effects of a single $20 \mathrm{mg}$ dose of letrozole on ovarian function post dominant follicle selection: an exploratory randomized controlled trial. J Ovarian Res. 2017;10:6.

16. Park YH, Kim TY, Kim GM, et al, Korean Cancer Study Group (KCSG). Palbociclib plus exemestane with gonadotropin-releasing hormone agonist versus capecitabine in premenopausal women with hormone receptor-positive, HER2-negative metastatic breast cancer(KCSG-BR15-10): a multicentre, open-label, randomised, phase 2 trial. Lancet Oncol. 2019;20(12):1750-9.

17. Huerta-Reyes M, Maya-Núñez G, Pérez-Solis MA, et al. Treatment of Breast Cancer With Gonadotropin-Releasing Hormone Analogs. Front Oncol. 2019;9:943.

18. Silvestris E, Dellino M, Cafforio P, et al. Breast cancer: an update on treatment-related infertility. J Cancer Res Clin Oncol. 2020;146(3):647-57.

19. Stuursma A, van Driel CMG, Wessels NJ, et al. Severity and duration of menopausal symptoms after risk-reducing salpingo-oophorectomy. Maturitas. 2018;111:69-76.

20. Hsieh AH, Kichenadasse G, Vatandoust S, et al. Goserelin toxicities and preferences for ovarian suppression method in pre-menopausal women with breast cancer. Intern Med J. 2016;46(10):1153-9.

21. Lan B, Jiang S, Li T, et al. Depression, anxiety, and their associated factors among Chinese early breast cancer in women under 35 years of age: A cross sectional study. Curr Probl Cancer. 2020; 100558.

22. Zhang $C$, Cui $L$, Zhang $L$, et al. Sexual activity and function assessment in middle-aged Chinese women using the female sexual function index. Menopause. 2017;24(6):669-76.

23. Xia J, Tang Z, Wu P, et al. Use of item response theory to develop a shortened version of the EORTC QLQ-BR23 scales. Sci Rep. 2019;9(1):1764.

24. Alshehre Sallwa M, Duffy Sheila,Jones Georgina, et al. A prospective, single-centre, single-arm, open label study of the long term use of a gonadotropin releasing hormone agonist (Triptorelin SR, 11.25 $\mathrm{mg}$ ) in combination with Tibolone add-back therapy in the management of chronic cyclical pelvic pain. Reprod Biol Endocrinol. 2020;18:28.

25. Zhang P, Li CZ, Jiao GM, et al. Effects of ovarian ablation or suppression in premenopausal breast cancer: A meta-analysis of randomized controlled trials. Eur J Surg Oncol. 2017;43(7):1161-72.

26. Park EM, Gelber S, Rosenberg SM, et al. Anxiety and Depression in Young Women With Metastatic Breast Cancer: A Cross-Sectional Study. Psychosomatics. 2018;59(3):251-8. 
27. Pooley AE, Benjamin RC, Sreedhar S, et al. Sex differences in the traumatic stress response: the role of adult gonadal hormones. Biol Sex Differ. 2018;9(1):32.

28. Mahmoud R, Wainwright SR, Chaiton JA, et al. Ovarian hormones, but not fluoxetine, impart resilience within a chronic unpredictable stress model in middle-aged female rats. Neuropharmacology. 2016;107:278-93.

29. Nguyen TV, Reuter JM, Gaikwad NW, et al. The steroid metabolome in women with premenstrual dysphoric disorder during GnRH agonist-induced ovarian suppression: effects of estradiol and progesterone addback. Transl Psychiatry. 2017;7(8):e1193.

30. Fugger G, Dold M, Bartova L, et al. Major Depression and Comorbid Diabetes - Findings from the European Group for the Study of Resistant Depression. Prog Neuropsychopharmacol Biol Psychiatry. 2019;94:109638.

31. Zhang Y, Chen Y, Ma L. Depression and cardiovascular disease in elderly: Current understanding. J Clin Neurosci. 2018;47:1-5.

32. Maciejewska-Jeske M, Szeliga A, Męczekalski B. Consequences of premature ovarian insufficiency on women's sexual health. Prz Menopauzalny. 2018;17(3):127-30.

33. Kuehn R, Casaubon J, Raker C, et al. Sexual Dysfunction in Survivorship; the Impact of Menopause and Endocrine Therapy. Ann Surg Oncol. 2019;26(10):3159-65.

34. Robinson PJ, Bell RJ, Christakis MK, et al. Aromatase Inhibitors Are Associated With Low Sexual Desire Causing Distress and Fecal Incontinence in Women: An Observational Study. J Sex Med. 2017;14(12):1566-74.

35. Gandhi C, Butler E, Pesek S, et al. Sexual Dysfunction in Breast Cancer Survivors: Is it Surgical Modality or Adjuvant Therapy? Am J Clin Oncol. 2019;42(6):500-6.

36. Stabile C, Goldfarb S, Baser RE, et al. Sexual health needs and educational intervention preferences for women with cancer. Breast Cancer Res Treat. 2017;165(1):77-84.

37. Hummel SB, Hahn DEE, van Lankveld JJDM, et al. Factors Associated With Specific Diagnostic and Statistical Manual of Mental Disorders, Fourth Edition Sexual Dysfunctions in Breast Cancer Survivors: A Study of Patients and Their Partners. J Sex Med. 2017;14(10):1248-59.

38. Soldera SV, Ennis M, Lohmann AE, et al. Sexual health in long-term breast cancer survivors. Breast Cancer Res Treat. 2018;172(1):159-66.

39. Flynn KE, Reese JB, Jeffrey D. Patient experiences with communication about sex during and after treatment for cancer. Psychooncology. 2012;21:594-601.

40. Bober SL, Fine E, Recklitis CJ. Sexual health and rehabilitation after ovarian suppression treatment (SHARE-OS): a clinical intervention for young breast cancer survivors. J Cancer Surviv. 2020;14(1):26-30.

41. Davis SR, Robinson PJ, Jane F, et al. Intravaginal Testosterone Improves Sexual Satisfaction and Vaginal Symptoms Associated With Aromatase Inhibitors. J Clin Endocrinol Metab. 2018;103(11):4146-54. 
42. Tong J, Zhang C, Zhang L, et al. Analysis of low sexual desire in Chinese women: a national population-based epidemiological survey in mainland China. Sex Health. 2020;17(1):61-8.

43. Li L, Yang B, Li H, et al. Chinese multicentre prospective registry of breast cancer patient-reported outcome-reconstruction and oncoplastic cohort (PRO-ROC): a study protocol. BMJ Open. 2019;9(12):e032945.

\section{Figures}

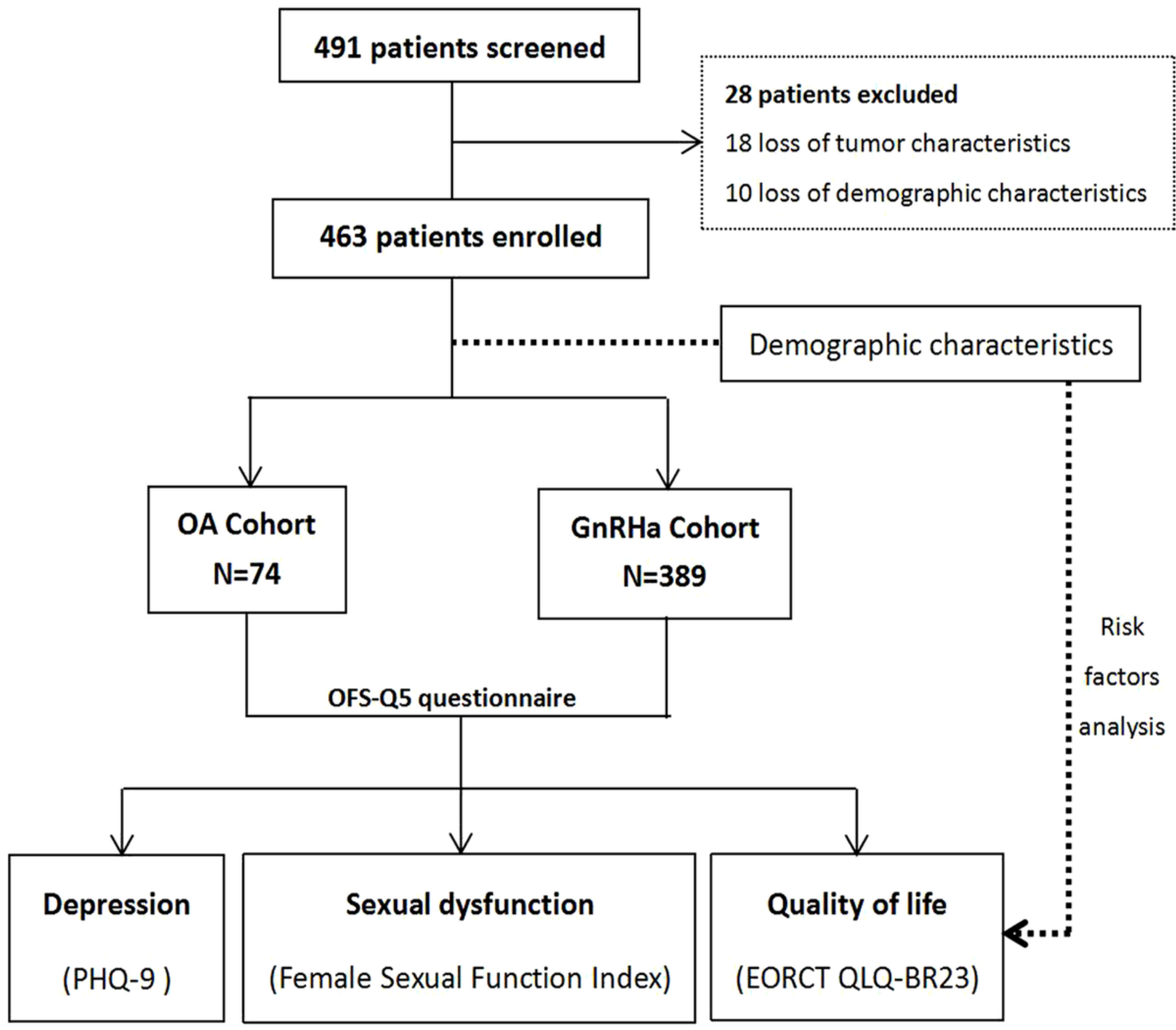

Figure 1

Patient disposition in our study Flow diagram showing patient enrollment, allocation and analysis in this study. OA, Ovarian Ablation; GnRHa, Gonadotropin releasing hormone (GnRH) agonist; OFS-Q5, Ovarian 
function suppression-Questionnaire 5; PHQ-9, Patient Health Questionnaire-9 items;

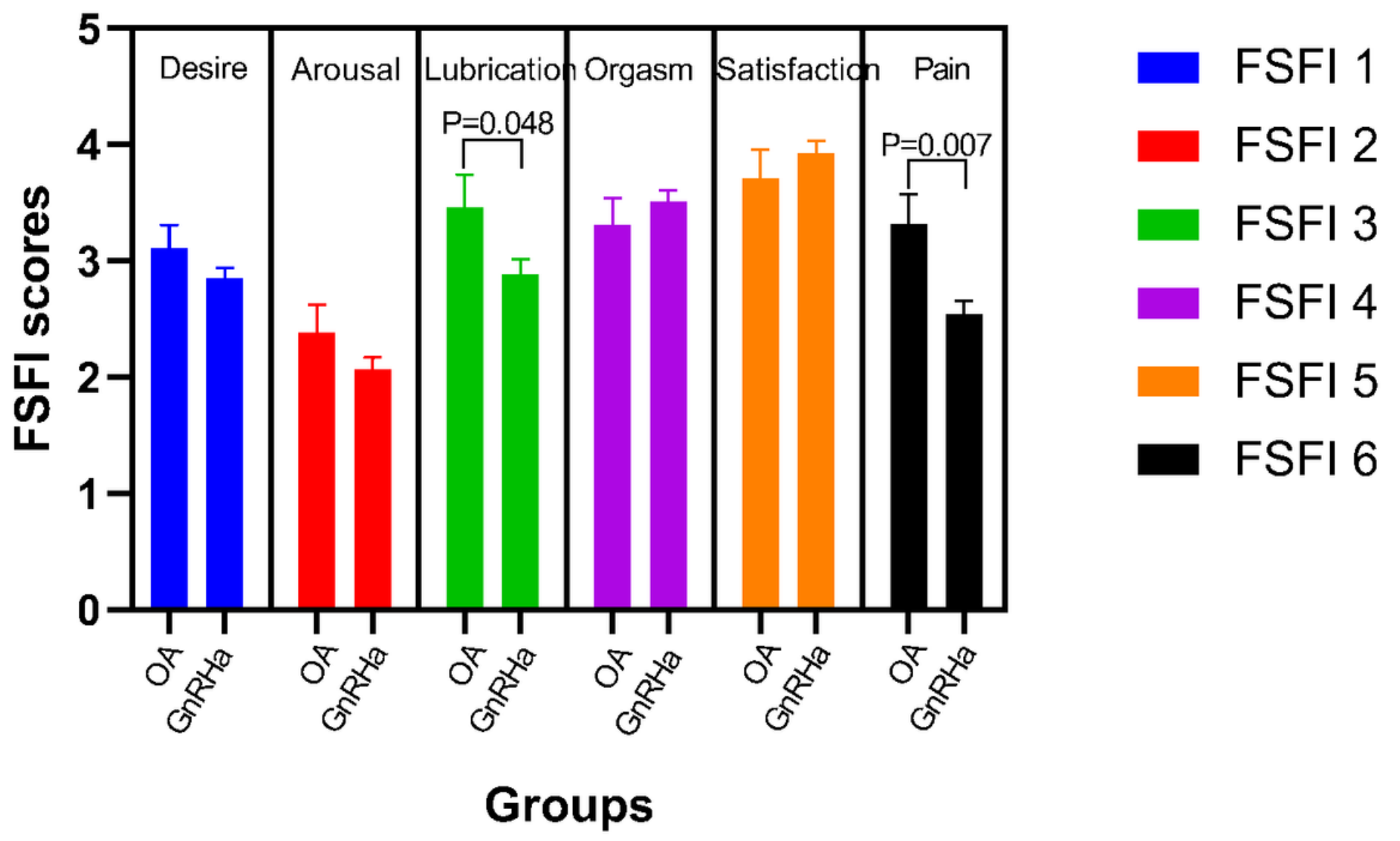

Figure 2

Sexual function sub-items between medicine GnRHa and ovarian ablation. FSFI 1: sexual desire; FSFI 2: sexual arousal; FSFI 3: sexual lubrication; FSFI 4: sexual orgasm; FSFI 5: sexual satisfaction; FSFI 6: sexual pain. OA: ovarian ablation; GnRHa: gonadotropin releasing-hormone agonists. 